\title{
Brazilian Experience of the Treatment of Alopecia Universalis with the Novel Antirheumatic Therapy Tofacitinib: A Case Series
}

\author{
Morton Scheinberg - Regina Adalva de Lucena Couto Océa • Boris Afonso Cruz • \\ Sineida Berbert Ferreira
}

Received: June 1, 2017 / Published online: July 28, 2017

(c) The Author(s) 2017. This article is an open access publication

\begin{abstract}
Introduction: Alopecia universalis is an autoimmune disorder for which there is no known effective therapy. Tofacitinib-a novel antirheumatic therapy for rheumatoid arthritis-has been shown in some reports to induce sustained hair growth in patients with alopecia universalis.

Case Series: Here, we review the experiences of four different rheumatologists across the country regarding four Brazilian patients with alopecia universalis who were treated with tofacitinib. Two of these four patients had idiopathic alopecia and the other two had rheumatoid arthritis; in each case, the alopecia
\end{abstract}

Enhanced content To view enhanced content for this article go to www.medengine.com/Redeem/ 12E8F0602FAC9A45.

M. Scheinberg $(\square)$

Hospital Israelita Albert Einstein, São Paulo, Brazil e-mail: morton@osite.com.br

M. Scheinberg

AACD Hospital, São Paulo, Brazil

R. A. de Lucena Couto Océa

Rheumatology Section Universidade Federal,

Sergipe, Brazil

B. A. Cruz

Biocor Hospital Belo Horizonte, Minas Gerais, Brazil

S. B. Ferreira

DermatoSineida Clinic Maringa, Paraná, Brazil universalis was apparently induced by etanercept.

Conclusion: Tofacitinib should be considered for the treatment of severe alopecia areata, but the optimal dose and duration of therapy should be defined in randomized controlled trials.

Keywords: Alopecia; Biologic therapy; Rheumatoid arthritis; Tofacitinib; Universalis

\section{INTRODUCTION}

Alopecia universalis (AU) is a variant of alopecia areata (AA) that can affect both children and adults and is associated with the complete loss of all body hair [1]. It is well known that the etiology of $\mathrm{AU}$ is related to a complex interaction between genetic and immune abnormalities that target the hair follicle. $T$ cells have been shown to be important effector cells in the appearance of $\mathrm{AU}$, as they express cytokinesparticularly interferon gamma-that target the hair follicle [2]. While several therapies for AU have been tested, they have all shown only partial or no clinical efficacy [3]-there are currently no effective therapies for AU. In the past 3 years, a few reports have been published suggesting that some AU patients appear to respond to Janus kinase inhibitors (JAKi), implying that JAKi should be evaluated as a promising novel therapy $[4,5]$. JAKi 
downregulate interferon expression. Given that the expression of interferon is known to be increased in $\mathrm{AU}$, this could explain the reported response of $\mathrm{AU}$ to JAKi [6]. In this paper, we report the short-term follow-up of four cases of $\mathrm{AU}$ in Brazil that remitted after the administration of tofacitinib. The individual aspects of each case are discussed and compared to the limited literature available on this topic at the present time.

\section{CASE SERIES}

Patient 1: Male, 38 years of age, who had developed AU 10 years earlier. The patient was initially treated with oral, topical, and intralesional corticosteroids followed by minoxidil, methotrexate, and cyclosporine, all with no response. He also presented with dystrophic fingernails. On examination, he showed a total absence of eyebrows, eyelashes, beard, hair on the trunk, scalp, genitals, arms, and legs. He was started on $5 \mathrm{mg}$ of tofacitinib twice a day-an off-label indication with prior patient consent. After 2 months he developed initial hair growth on the scalp, and there was progressive hair growth after 9 months of therapy (Fig. 1). Currently, the hair is still growing despite a reduction in the dose to $5 \mathrm{mg}$ a day [5].

Patient 2: Female, 20 years of age, who had developed $\mathrm{AU}$ at age ten and had tried several treatments, including oral and intralesional steroids, methotrexate, and cyclosporine, all with no detectable response. After 6 weeks of therapy with tofacitinib $5 \mathrm{mg}$ twice a day, it was possible to observe partial growth of scalp, eyebrow, and axillary hair (Fig. 2).

Patient 3: Male, 45 years of age, who had developed rheumatoid arthritis 7 years earlier. The patient was treated initially with leflunomide, then prednisone $5 \mathrm{mg}$ for 2 years, which was followed by etanercept, leading to clinical remission of the disease. Two years ago the patient presented with diffuse pruritus, erythematous skin, and initial hair loss on the eyebrows. Leflunomide was initially discontinued and a conventional antiallergic medication was administered but the skin disease persisted. Etanercept was discontinued and abatacept was introduced, but the joint disease remained active and the clinical picture for the skin evolved to alopecia universalis. Tofacitinib was started at $5 \mathrm{mg}$ twice a day, and hair growth appeared on the scalp, eyebrows, and skin after 7 months (Fig. 3).

Patient 4: Female, 60 years of age, who had developed seropositive rheumatoid arthritis 2 years earlier. The patient was in remission due to treatment with etanercept and methotrexate when progressive hair loss developed. All medications were withdrawn; subsequent treatment with oral prednisone and intralesional triamcinolone injections had no effect. She progressed to universal alopecia and her arthritis became active again. Tofacitinib was introduced, and 6 months after that the patient's arthritis had improved and the alopecia had fully resolved (Fig. 4).

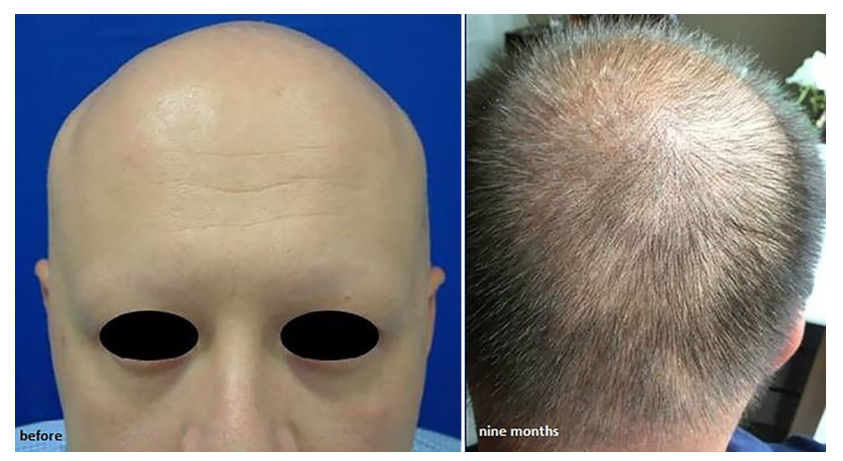

Fig. 1 Patient 1: before and 9 months after the introduction of tofacitinib $10 \mathrm{mg}$ daily 


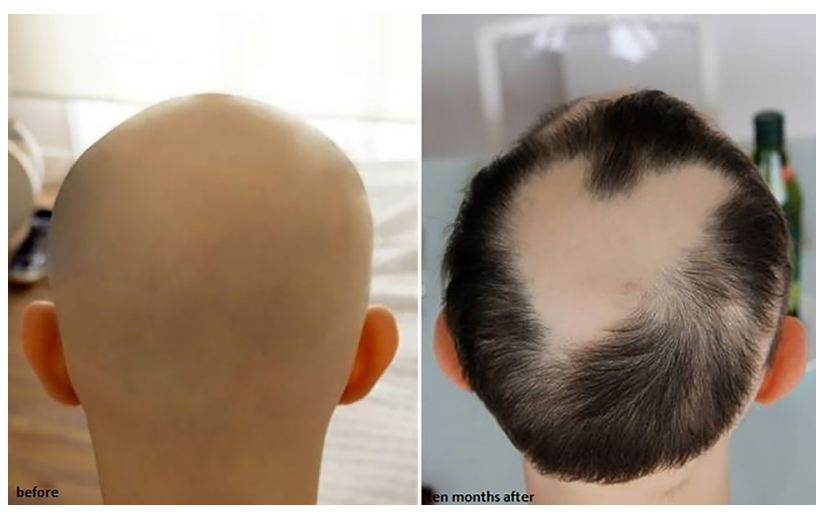

Fig. 2 Patient 2: before and 10 months after the introduction of tofacitinib $10 \mathrm{mg}$ daily

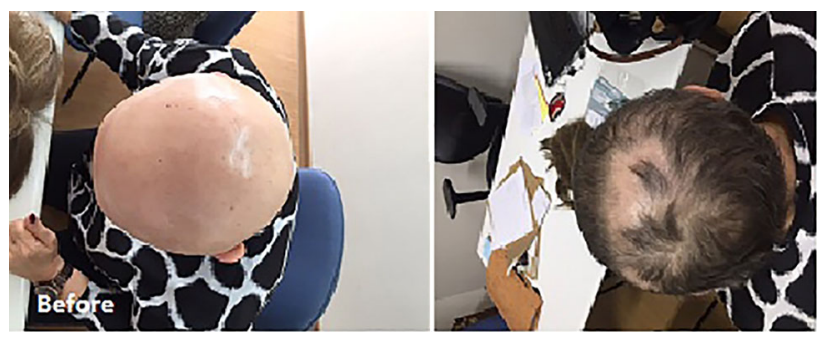

Fig. 3 Patient 3: hair growth after 7 months of tofacitinib $10 \mathrm{mg}$ daily

Hair growth after $10 \mathrm{mg}$ of Tofacitinib daily

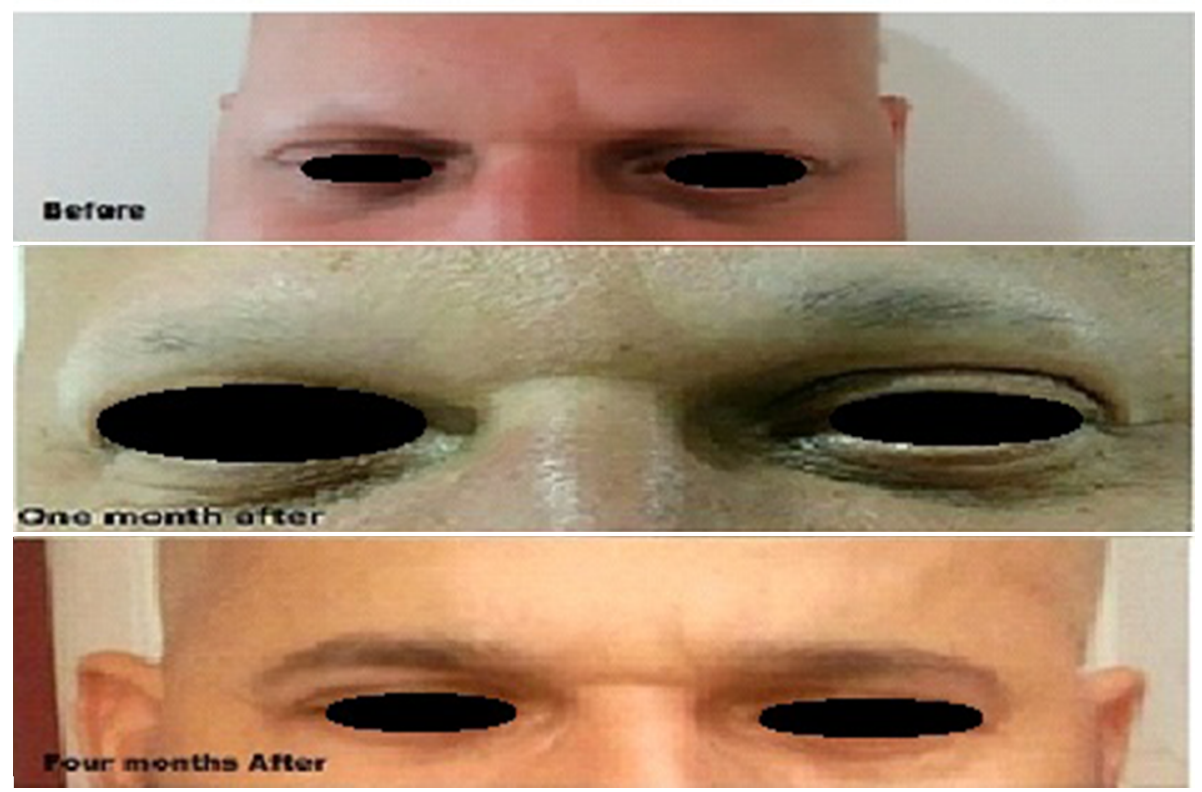

Fig. 4 Patient 4: hair growth 1 and 4 months after the introduction of tofacitinib $10 \mathrm{mg}$ daily 
This case series complied with the ethical standards of the AACD Hospital. All procedures followed the ethical standards for human experimentation, and informed consent was obtained from all patients.

\section{DISCUSSION}

Tofacitinib citrate is a small molecule that selectively inhibits Janus kinase 1 and 3 and was approved for the treatment of moderate to severe rheumatoid arthritis [7]. Since it affects various $\mathrm{T}$ cell effector functions, it is also being evaluated for the treatment of other autoimmune inflammatory conditions of the skin, such as psoriasis and atopic dermatitis. Alopecia areata and universalis are considered to be autoimmune in their pathogenesis due to the attack of $T$ cells on hair follicles, which leads to transient or permanent nonscarring hair loss [8]. Recently, new molecular pathways that are involved in AA were discovered, including autophagy/apoptosis, transforming growth factor/Tregs, and Janus kinase signaling [6]. In this paper, we have presented four Brazilian patients who developed progressive hair growth upon treatment with tofacitinib at the recommended dose approved for the treatment of rheumatoid arthritis. All four cases had a clinical diagnosis and no biopsy was performed in any of the patients. There are very few reports on this topic in the literature. The first was from the Yale Dermatology Group, who reported that tofacitinib appeared to "kill two birds with one stone:" alopecia was reversed in a patient with psoriasis [9]. That was followed by the largest report yet from the same group-Liu and coworkers reported on 90 patients who had alopecia areata or variants of it. These results are summarized in Table 1.

In the report by the Yale group, almost $60 \%$ of the patients showed a $>50 \%$ change in the alopecia score (SALT severity of alopecia score tool), which was sustained for variable periods of time.

The duration of the response varied from 4 to 18 months. The lack of a control group makes the conclusions of the study somewhat limited, but the results do appear to confirm those given in other isolated reports from a few groups that are available in the literature [10]. In one of the reported cases, initial signs of hair regrowth were observed in the same timeframe as reported by Azengruber and coauthors, but the efficacy of the drug quickly waned again, leading to a relapse of the disease [11]. In a recently released report, the authors conducted a review and suggested that hair growth following tofacitinib treatment may be partial and transient for some patients [12]. In our cases, the efficacy of tofacitinib persisted; indeed, reducing the dose by half in one of those cases did not affect the positive response (see Fig. 1).

Table 1 Clinical response of patients with alopecia to tofacitinib

\begin{tabular}{lll}
\hline $\begin{array}{l}\text { Magnitude of clinical response } \\
\text { SALT score }\end{array}$ & Percentage of patients (\%) & Number of patients \\
\hline Complete response (>90\%) & 20 & 13 \\
Intermediate response (51-90\%) & 38.4 & 25 \\
Moderate response (6-50\%) & 18.5 & 12 \\
No response (<5\%) & 23.1 & 15 \\
\hline Therapy & Percentage of patients (\%) \\
\hline Standard monotherapy. Tofacitinib 5 mg BID & 43.1 & Number of patients \\
Adjuvant therapy & 56.9 & 25 \\
Tofacitinib >5 mg BID & 29.2 & 37 \\
Tofacitinib 5 mg BID + prednisone & 13.8 & 19 \\
\hline
\end{tabular}


Thus, even though tofacitinib is a promising new treatment option, we need to learn more about its potential, including the appropriate duration of treatment, the maintenance dose, the possibility of relapse if the drug is discontinued, and the loss of response during treatment, as described in one of the reports in the literature. None of our patients developed adverse events, but it is important to monitor for such effects (including infections and hyperlipidemia) during long-term therapy.

Patient 3 in this report developed alopecia areata during anti-TNF treatment. A few such cases have been reported previously $[13,14]$. It is worth noting that the induction of other autoimmune diseases by prolonged anti-TNF treatment has been extensively reported in the medical literature $[13,14]$.

The possible application of JAKi to other autoimmune disorders such as psoriasis and inflammatory bowel disease is currently being researched [15]. Nail dystrophy is a heterogeneous skin condition, but in some subtypes its development has autoimmune origins. We recently reported that tofacitinib appears to be beneficial in patients with this nail disorder, which can also be associated with other rheumatic disorders such as psoriatic arthritis and is observed in approximately $50 \%$ of AU patients [16].

The long-term safety of tofacitinib in patients with rheumatic disease will be closely monitored in years to come, as will its long-term safety in patients with AU. Tofacitinib is similar to biologics in that long-term use can lead to opportunistic infections, in particular those associated with persistent viral infections. In such cases vaccination can help patients who require the long-term use of tofacitinib [17].

\section{CONCLUSIONS}

In conclusion, targeting the intracellular JAK signaling pathway appears to be a promising therapeutic strategy for AA patients, and the future should bring new information along with the advent of second-generation selective JAK inhibitors which are currently being investigated. It is expected that prospective randomized controlled trials will be performed to determine the real efficacy of tofacitinib in patients with AA only or AA in association with other rheumatic diseases.

\section{ACKNOWLEDGEMENTS}

No sponsorship or funding was received for this study, and the article processing charges were funded by the authors. All of the named authors meet the International Committee of Medical Journal Editors (ICMJE) criteria for authorship for this manuscript, take responsibility for the integrity of the work as a whole, and gave their final approval for the version to be published.

Disclosures. Morton Scheinberg, Regina Océa, Boris Cruz, and Sineida Ferreira have nothing to disclose.

Compliance with Ethics Guidelines. All procedures followed the ethical standards for human experimentation, and informed consent was obtained from all patients.

Open Access. This article is distributed under the terms of the Creative Commons Attribution-NonCommercial 4.0 International License (http://creativecommons.org/licenses/ by-nc/4.0/), which permits any noncommercial use, distribution, and reproduction in any medium, provided you give appropriate credit to the original author(s) and the source, provide a link to the Creative Commons license, and indicate if changes were made.

\section{REFERENCES}

1. Hordinsky MK. Overview of alopecia areata. J Investig Dermatol Symp Proc. 2013;16:S513-5.

2. Gilhar A, Paus R, Kalish RS. Lymphocytes, neuropeptides, and genes involved in alopecia areata. J Clin Invest. 2007;117:2019-27.

3. Dainichi T, Kabashima K. Alopecia areata: what's new in epidemiology, pathogenesis, diagnosis, and therapeutic options? J Dermatol Sci. 2017;86:3-12 (Review). 
4. Crispin MK, Ko JM, Craiglow BG, Cerise JE, et al. Safety and efficacy of the JAK inhibitor tofacitinib citrate in patients with alopecia areata. JCI Insight. 2016;22(1):e89776.

5. Scheinberg M, Ferreira SB. Reversal of alopecia universalis by tofacitinib: a case report. Ann Intern Med. 2016;15(165):750-1.

6. Samadi A, Ahmad Nasrollahi S, Hashemi A, Nassiri Kashani M, Firooz A. Janus kinase (JAK) inhibitors for the treatment of skin and hair disorders: a review of literature. J Dermatol Treat. 2017;22:1-11.

7. Fleischmann R, Mease PJ, Schwartzman S, et al. Efficacy of tofacitinib in patients with rheumatoid arthritis stratified by background methotrexate dose group. Clin Rheumatol. 2017;36(1):15-24.

8. Rork JF, Rashighi M, Harris JE. Understanding autoimmunity of vitiligo and alopecia areata. Curr Opin Pediatr. 2016;28:463-9.

9. Craiglow BG, King BA. Killing two birds with one stone: oral tofacitinib reverses alopecia universalis in a patient with plaque psoriasis. J Invest Dermatol. 2014;134:2988-90.

10. Liu LY, Craiglow BG, Dai F, King BA. Tofacitinib for the treatment of severe alopecia areata and variants: a study of 90 patients. J Am Acad Dermatol. 2017;76:22-8.
11. Anzengruber F, Maul JT, Kamarachev J, Trüeb RM, French LE, Navarini AA. Transient efficacy of tofacitinib in alopecia areata universalis. Case Rep Dermatol. 2016;22(8):102-6.

12. Kassira S, Korta DZ, Chapman L, Dann F. Review of treatment for alopecia totalis and alopecia universalis. Int J Dermatol. 2017. (epub ahead of print).

13. Bene J, Moulis G, Aufrett M, et al. Alopecia induced by tumor necrosis factor: description of 52 cases and disproportionally analysis in a nationwide pharmacovigilance database. Rheumatology. 2014;53:1465-9.

14. Laurindo IM, Scheinberg M. Why do some biological agents induce psoriasis or psoriasiform lesions? Nat Clin Pract Rheumatol. 2008;4:168-9.

15. Banerjee S, Biehl A, Gadina M, Hasni S, Schwartz DM. JAK-STAT signaling as a target for inflammatory and autoimmune diseases: current and future prospects. Drugs. 2017;77:521-46.

16. Jaller JA, Jaller JJ, Jaller AM, et al. Recovery of nail dystrophy potential new therapeutic indication of tofacitinib. Clin Rheumatol. 2017;36:971-3.

17. Winthrop KL. The emerging safety profile of JAK inhibitors in rheumatic disease. Nat Rev Rheumatol. $2017 ; 13: 234-43$. 